\title{
One-step fabrication of polymer components for microphotonics by gray scale electron beam lithography
}

L. Dong

lindong@kth.se

\section{S. Popov}

\section{A.T. Friberg}

Royal Institute of Technology, Division of Optics, Electrum 229, 16440 Kista, Sweden

Royal Institute of Technology, Division of Optics, Electrum 229, 16440 Kista, Sweden

Royal Institute of Technology, Division of Optics, Electrum 229, 16440 Kista, Sweden Aalto University, Department of Applied Physics, PO Box 13500, 00076 Aalto, Finland University of Eastern Finland, Department of Physics and Mathematics, PO Box 111, 80101 Joensuu, Finland

We demonstrate an application of gray scale electron beam lithography (EBL) for the fabrication of polymer waveguides and grating output couplers with depth variable features, using the SU-8 resist. The technique is mainly applicable for multi-level binary profile, where groove depths of the structure are controlled by choosing a proper exposure dose. Unlike reactive ion etching which is limited by the lag effect, the gray scale EBL allows free combination of groove widths and depths. Shrinking effect which is critical in polymer couplers' writing is taken into account and can be compensated. For better fabrication feasibility, the grating couplers can be simultaneously produced with waveguides with no inter-step alignment required. Therefore, this is a promising technique in manufacturing grating output couplers for polymer based waveguides with high performance in terms of mode matching/confinement and coupling efficiency. [DOI: 10.2971/jeos.2011.11010]

Keywords: gray scale electron beam lithography, 3D polymer waveguides and grating couplers, lag effect

\section{INTRODUCTION}

Microscale optical devices for "Lab-on-a-chip" (LOC) application, like waveguides and micro-cavities, using polymer materials, have been intensively developed due to favorable optical properties, flexible morphology and low material cost [1]-[3]. Certain types of output coupling scheme are exploited to couple light signals from device to fiber or detector. As a typical solution, grating output coupler can propagate the light out of the substrate plane into arbitrary direction with specially designed grating profile. This allows avoiding cutting the substrate and polishing the facet that is required for some other schemes like butt coupling [1]. Thus, grating output couplers have become the mainstream in the area and numerous investigations to optimize the fabrication of such design were inspired.

Typical grating output couplers giving high coupling efficiency include the shallow-etched grooves [4], metal lines [5], curved and chirped gratings [6] and slanted grooves [7]. Although most of these designs are based on a silicon-oninsulator (SOI) technique, they can be adopted to a polymer based platform with merely geometrical size modification. Several aspects are in the focus of discussion while translating technology suitable for SOI platform to polymer based technique.

Application of the EBL for electron sensitive polymer materials offers an obvious advantage, simplicity of manufacturing. Here the primary pattern (which is only a first step in SOI fabrication process) defined by lithography is the final functioning device. No inter-step alignment is needed for such one-batch processes. This could enhance the accuracy and increase the fabrication speed considerably in a research environment where the structure design is modified frequently and fast prototyping of single structure is required. Moreover, with such fabrication method, it is more likely to get smooth and sharp sidewalls compared with reactive ion etched SOI devices. On the other hand, due to lower refractive index contrast, the coupling efficiency of a polymer grating is not as high as in SOI grating. In practice, this feature leads to longer grating length to provide good coupling efficiency. Long polymer gratings with nano-sized features can suffer from the shrinking effect which should be properly compensated.

In comparison with standard use of the EBL which is mostly used for making patterns of the same depth (binary levels), it is possible to directly make three dimensional polymer structures with gray scale EBL [8] using the unsaturated dose area of a resist which has a low contrast, like SU-8 $[9,10]$. As reported in this work, the dimension in the vertical direction (the groove depth of a grating) is independent of that in the horizontal direction (the groove width) with this technique, 
i.e. we observe no lag effect (correlation between the depth and width of grating grooves). With gray scale EBL, it can be avoided by using variable exposure dose. This provides certain freedom to write grooves with arbitrary depths and widths, which can help improving the coupling efficiency and mode confinement [11]. This is an obvious advantage in comparison with reactive ion etching (RIE) process which leads to the lag effect deteriorating the performance of a final device [12].

Similar applications with another resist, PMMA, have been demonstrated [13]. However, the large exposure dose for PMMA (usually 50-500 $\mu \mathrm{C} / \mathrm{cm}^{2}$, compared with a dose less than $3 \mu \mathrm{C} / \mathrm{cm}^{2}$ for SU-8) does not make it suitable for the cases in which active media like dye or quantum dots are doped in the polymer. The high energy of the electrons is likely to deteriorate the functionality of the dopants or even bleach them. On the other hand, a good example which involves dye doped SU-8 distributed feedback laser was demonstrated by Balslev et al. [14]. In that case, grating with $200 \mathrm{~nm}$ pitch and $10 \mathrm{~nm}$ depth was fabricated by gray scale EBL. It shows the feasibility of the gray scale EBL for direct fabrication of SU-8 devices.

Even though polymer gratings with fixed depth made by gray scale EBL have been reported [13, 14], much less is known about performance of gray scale EBL (groove depth/ width dependence, fabrication accuracy etc.) in fabricating polymer gratings with variable groove depths. The potential of this technique to manufacture grating couplers with even better performances in terms of coupling efficiency and mode confinement requires deeper understanding. In this work, we investigate the application of the grey scale EBL for fabrication of three dimensional grating output coupler from SU-8. We show possibility for creating a variable groove depth profile rather than the fixed depth for the grating [14], to achieve better functionalities [11]. In our study, we identify the optimum dose range in which the post-development resist thickness is nearly linearly dependent on the electron dose, and the suitable dose value for waveguide writing is defined. Next, it is found that structures with lower dose tend to shrink after development, provided that the feature size of the structure is small and the structure is rather isolated. We have also fabricated several prototypes of $10 \mu \mathrm{m}$ wide polymer waveguide with gratings on it. The pitch of the gratings is $1.3 \mu \mathrm{m}$ with a filling factor of $50 \%$ which are suitable for working in the telecom band. An aspect ratio of more than 0.4 can be achieved. We demonstrate that the groove depth is independent of the groove width, and no lag effect as with the RIE etching. The groove depth can be variably tuned from positive value to the negative (convex ribs). This technique is promising to give 3D polymer grating output couplers with modulated groove depth and high coupling efficiency.

\section{EXPERIMENTAL DETAILS}

In the beginning of the process, a thin $\mathrm{SiO}_{2}$ film with a thickness of $1.4 \mu \mathrm{m}$ is grown on the top of silicon substrate by plasma-enhanced chemical vapor deposition to act as the cladding layer for the polymer waveguide. The substrate is baked on a hot plate in $160{ }^{\circ} \mathrm{C}$ for 10 minutes to get rid of the moisture and make good stickiness between $\mathrm{SiO}_{2}$ and polymer photoresist. EBL resist SU-8 2002 (MicroChem, US) is mixed with cyclopentanone with volume ratio 1:0.3 to decrease the viscosity. The mixture is then spin-coated onto the substrate at the speed of $500 \mathrm{rpm}$ for 5 seconds and subsequently $2100 \mathrm{rpm}$ for 30 seconds, which gives a film thickness of $1.2 \mu \mathrm{m}$. Then a 1 minute pre-bake at $95^{\circ} \mathrm{C}$ is taken to evaporate the solvent.

Electron beam exposure is carried out with a 'Raith Turnkey 150' SEM and EBL system. An electron acceleration voltage of $25 \mathrm{kV}$ is chosen for all the exposures. The electron acceleration voltage is a key parameter to control forward and back scattering, the two types of scattering of electrons in the resist and substrate. Increasing the electron acceleration voltage usually intensifies the back scattering with simultaneous inhibiting the forward scattering, and vice versa. The forward scattering, an inelastic collision between incoming electrons and electrons in the resist/substrate material, has an effect on the effective beam diameter $d_{f}$ according to the empirical formula [15]:

$$
\mathrm{d}_{\mathrm{f}}=0.9\left(\mathrm{R}_{\mathrm{t}} / \mathrm{V}_{\mathrm{b}}\right)^{1.5}
$$

where $R_{t}$ is the thickness of the resist in nanometer and $V_{b}$ is the electron acceleration voltage in $\mathrm{kV}$. For a resist thickness larger than $1 \mu \mathrm{m}$ (as in this work), a relatively high electron acceleration voltage is required to get a small $d_{f}$ for writing the detailed features. On the other hand, the electron acceleration voltage should not be too high in a risk of incurring excessive back scattering which represents the elastic collision of incoming electrons with material nucleus and causes the proximity effect. The proximity effect can induce vital size and shape errors in periphery of the structures. Therefore, a moderate electron acceleration voltage of $25 \mathrm{kV}$ is chosen, provided the commonly used voltage value ranges from 5 to $100 \mathrm{kV}$. As shown later, this voltage did result in clear and sharp sidewalls of the waveguides with an appropriate exposure dose. Another important parameter of the EBL is the aperture size of the electron beam. It determines to a large extent the precision of exposure for small features. Here we keep the aperture size of $10 \mu \mathrm{m}$, almost the smallest one possible with this machine, to attain the ability of detailed writing. We use electron dose ranging from 0.5 to $3 \mu \mathrm{C} / \mathrm{cm}^{2}$ in order to get a gray scale response of the SU-8 resist. This range is much smaller than those of other EBL resists like PMMA, ma-N 2405 etc, making SU-8 competitive for time efficient EBL writing. After the exposure, different post exposure bake (PEB) temperatures, in particular $45^{\circ} \mathrm{C}$ and $105^{\circ} \mathrm{C}$, were applied to obtain various remaining thickness and resist contrast. All the PEBs were performed for 1 minute. After that, the patterns were developed with standard SU-8 developer from MicroChem for 2 minutes, rinsed in 2-propanol for 10 seconds and blown dry with nitrogen. 
a)

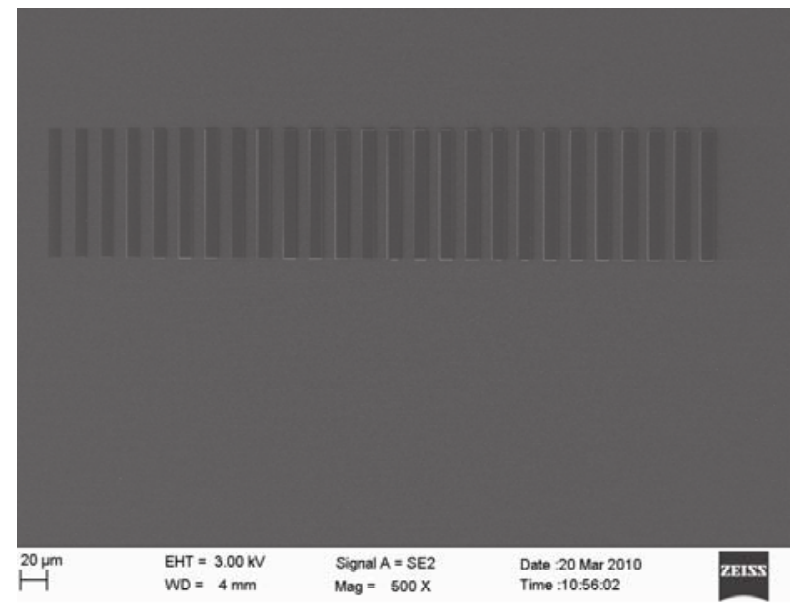

b)

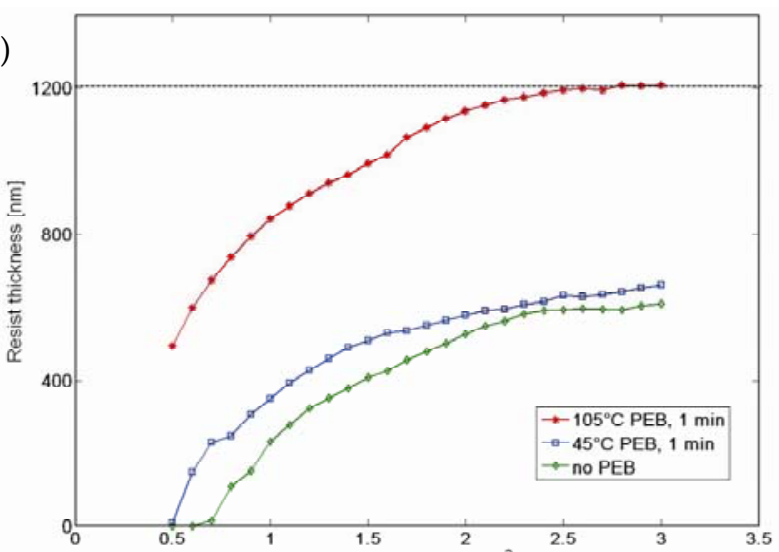

FIG. 1 a) SEM image of the dose variation for SU-8 bars with $10 \mu \mathrm{m}$ width and $10 \mu \mathrm{m}$ gap. b) SU-8 film thickness versus the electron beam exposure dose for different PEB conditions. The dashed black line shows the film thickness before exposure.

\section{RESULTS AND DISCUSSION}

\subsection{SENSITIVITY AND CONTRAST TEST}

Figure 1a) shows 26 SU-8 bars with linearly increased dose from 0.5 to $3 \mu \mathrm{C} / \mathrm{cm}^{2}$. This is a typical pattern used for dose response test of photoresist. The width of each bar is $10 \mu \mathrm{m}$ and the gap between adjacent bars is $10 \mu \mathrm{m}$. The remaining resist thickness with different $\mathrm{PEB}$ conditions is plotted in Figure 1b). One can see that for the dose range suitable for groove depth control of 3D gratings, the remaining thickness increases almost linearly while exposure dose is changed from 1 to $2 \mu \mathrm{C} / \mathrm{cm}^{2}$. The remaining thickness reaches the original thickness around $3 \mu \mathrm{C} / \mathrm{cm}^{2}$ for $105{ }^{\circ} \mathrm{C}$ PEB, while it is only half of the original thickness for the same dose with lower PEB temperatures. Thus, a lower PEB temperature gives a lower resist contrast which is a merit for gray scale writing. However, in other experiments, we use the $105{ }^{\circ} \mathrm{C} \mathrm{PEB}$ for all the tests in order to keep the dose as small as possible and, meanwhile, have the largest differences in the remaining thickness. Figure 2 gives a close view on the bars under high PEB temperature $\left(105^{\circ} \mathrm{C}\right)$. We can see that for the low dose range, the width of the bar increases with the dose (Figures $2 a$ ) and 2b)) until $1.6 \mu \mathrm{C} / \mathrm{cm}^{2}$, where the width reaches the nominal value of $10 \mu \mathrm{m}$ (Figure 2c). After that, overexposure occurs and the border of the bars loses its sharpness (Figure 2d)). Therefore, the dose for the $1.2 \mu \mathrm{m}$ thick waveguide writing should not exceed $1.6 \mu \mathrm{C} / \mathrm{cm}^{2}$ to ensure sharp sidewalls.

It is worth to notice that SU-8 is an extremely sensitive photoresist. It needs less than $3 \mu \mathrm{C} / \mathrm{cm}^{2}$ to be sufficiently exposed, compared to other commonly used resists, such as ZEP520A $\left(60 \mu \mathrm{C} / \mathrm{cm}^{2}\right)$, ma-N $2405\left(120 \mu \mathrm{C} / \mathrm{cm}^{2}\right)$ and PMMA $\left(50-500 \mu \mathrm{C} / \mathrm{cm}^{2}\right)$. This can be substantial merit when active media like dye or quantum dots are doped in the polymer structure. The high sensitivity of the resist, and low exposure dose as a consequence, helps to prevent the active media from being deactivated by large electron energy.

\subsection{SHRINKAGE OF WIDTH IN 3D STRUCTURES}

Another significant feature of the gray scale EBL is that exposure dose influences not only the remaining thickness, but also the footprint feature size in the substrate plane. Shrinkage is found with low dose. This effect can be easily understood concerning the negative tone feature of SU-8. With a low dose, electrons fail to spread into the edge of the patterns and it renders the resist to be underexposed. Meanwhile, this effect should not be ignored for waveguide and grating coupler applications where geometrical size is directly related to mode profile and coupling efficiency. For the $105{ }^{\circ} \mathrm{C}$ PEB condition, it is noted that the boundary between underexposure and overexposure lies around $1.5-1.6 \mu \mathrm{C} / \mathrm{cm}^{2}$ where the remaining thickness has not fully reached the original film thickness (only $80 \%$ ). Therefore, it is better to use an original film thickness larger than the required thickness in real applications to compensate the shrinkage with simultaneous preventing from overexposure.

The existence of shrinking effect may bring considerable errors to microstructures, especially to those with small feature sizes. Figure 3a) shows a 4-period 3D grating with high dose bars (HDB) and low dose bars (LDB) interleaving. The dose for HDB and LDB are 2.4 and $0.5 \mu \mathrm{C} / \mathrm{cm}^{2}$, respectively. The nominal bar widths for both are $650 \mathrm{~nm}$. The temperature of $105{ }^{\circ} \mathrm{C}$ for PEB is applied. An actual width obtained for the HDB $(665 \mathrm{~nm})$ is close to the nominal value, which is consistent to that shown in Figure 2. However, the width for the LDB is only $441 \mathrm{~nm}$, just $68 \%$ of the nominal value due to the shrinking effect. Faultages left by shrinkage can be clearly seen (inset of Figure 3a)). Shrinkage is also found in the direction along the bar. Another 4-period 3D grating with the same dose profile but wider bar width $(2 \mu \mathrm{m})$ is shown for comparison (Figure 3b)). The shrinkage effect is found to be less pronounced in larger structures (only 10\% shrinkage in the LDB).

The minimum impact of shrinking on such components can be clearly seen in Figure 4. Here we demonstrate one of two 

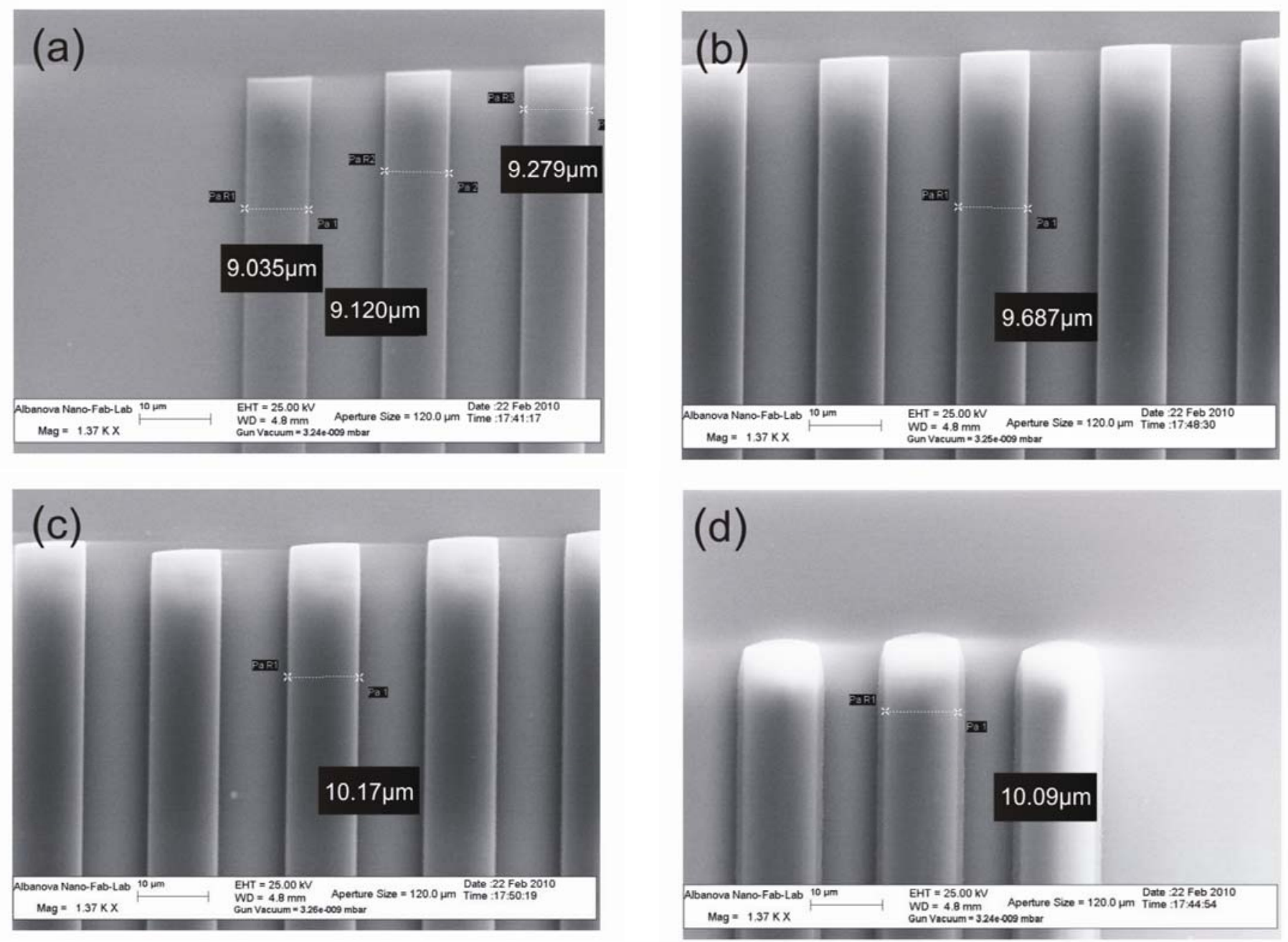

FIG. 2 Closer SEM images of SU-8 bars (shown in Figure 1 ) with different doses $\left(\mu \mathrm{C} / \mathrm{cm}^{2}\right)$ : a) 0.5-0.7; b) 1.0-1.4; $\mathrm{C}$ ) 1.4-1.8; d) $2.8-3.0$. The PEB temperature is $105^{\circ} \mathrm{C}$. The measured bar widths are shown in the images.

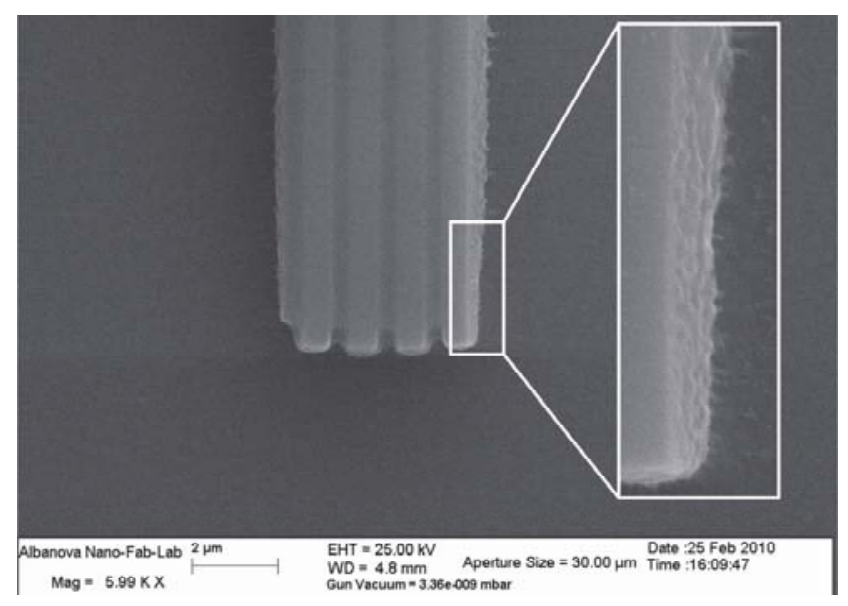

a)

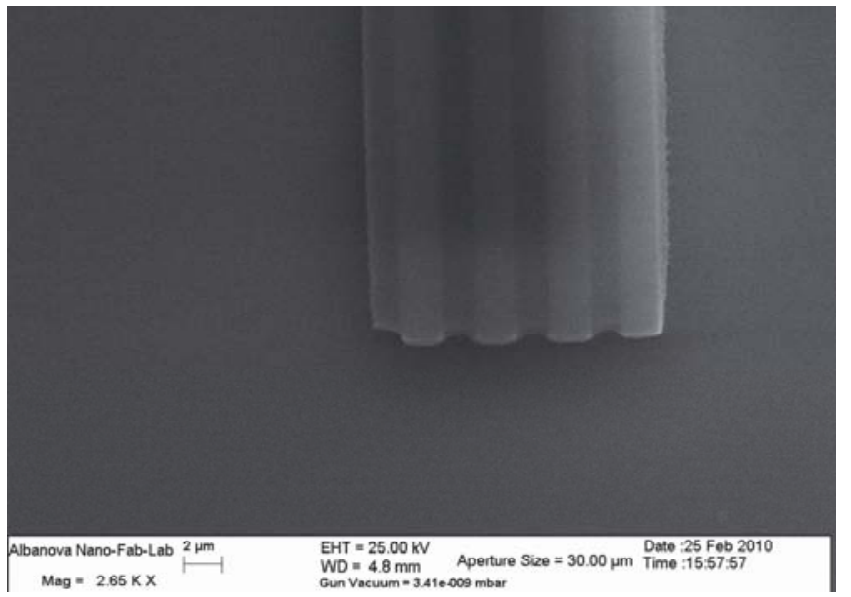

b)

FIG. 3 SEM images of 4-period SU-8 gratings with a) $1.3 \mu \mathrm{m}$ and b) $4 \mu \mathrm{m}$ pitch. The nominal groove widths are half of the pitch size for both. Severe shrinkage is observed in a); the obtained groove width is only $68 \%$ of the nominal value. The inset in a) shows the resist layers left by shrinking. The shrinkage is less prominent in b) with a larger feature size; only $10 \%$ groove width shrinking is found.

10-period 3D gratings, i.e. with controllable groove depth, which serve as input and output couplers for a long waveguide with $10 \mu \mathrm{m}$ width. The SEM images taken from the top and from a tilted angle are shown in Figures 4a) and b), respectively. The exposure dose for the groove area is $0.5 \mu \mathrm{C} / \mathrm{cm}^{2}$, and the dose for the main part of the waveguide is $2.4 \mu \mathrm{C} / \mathrm{cm}^{2}$. 


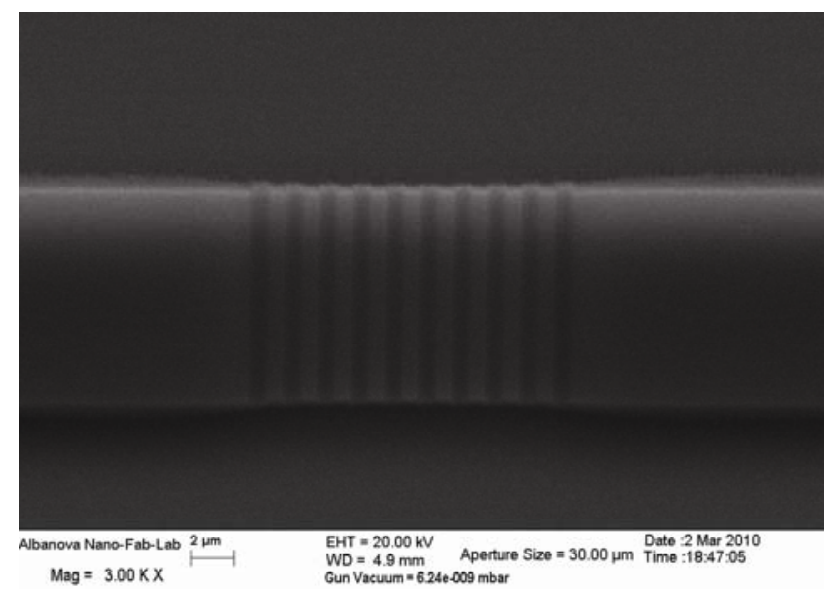

a)

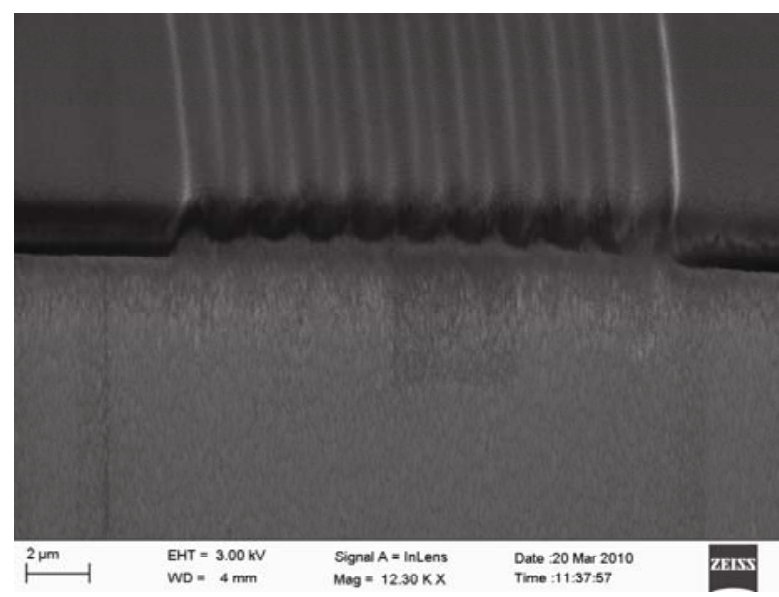

b)

FIG. 4 SEM images from a) top and b) a tilted angle of a 10-period SU-8 grating with fixed groove depth on a waveguide. The dose for the groove is $0.5 \mu \mathrm{C} / \mathrm{cm}^{2}$ and for the waveguide is $2.4 \mu \mathrm{C} / \mathrm{cm}^{2}$.

Although $2.4 \mu \mathrm{C} / \mathrm{cm}^{2}$ is not an optimized dose for waveguide writing as discussed before, it may yet be accepted for demonstration purposes of the gray scale EBL. This time, no obvious shrinkage in the longitudinal direction of the structure is found.

The shrinkage in the in-plane directions (i.e. the longitudinal direction and the transversal direction) comes most probably from the cross-linking of SU-8 [16]. This effect is especially distinct for isolated structures with a small feature size, as in Figure 3a). For structures embedded in large exposed area (Figure 4), however, the shrinking can be counteracted by the stress from the cross-linking of the large area (i.e. the waveguides on both sides of the grating in this case) and thus the size of the obtained structure is more close to the nominal value. It is even better if the large exposed area is symmetrically distributed around the structure in order to equalize the stresses from both sides. Therefore, in practice, it is advised to have symmetrical building blocks on both sides of a directly written grating. Alternatively, one can consider having larger original size to compensate the shrinking effect. Remarkably, the shrinking effect shows up anyway in the transverse direction that can be seen as slightly shorter width of the groove area in comparison with the waveguide. A factor of 1.1 in this direction has been applied to the grooves to successfully compensate the shrinkage. It is worth noting that the proximity effect (discussed in Section 2) may also induce dimension error in EBL defined SU-8 structures besides the cross-linking of SU-8. The difference is that the proximity effect influences mostly the size in the vertical direction rather than the in-plane directions. Due to the proximity effect, the thickness of the waveguide in Figure 4 turns out to be $9 \%$ higher than the thickness of the HDB in Figure 3a) even though they were assigned the same electron dose.

\subsection{COMPENSATING FOR LAG EFFECT AND 3D GRATING WITH VARIABLE GROOVE DEPTH}

In this section, we first apply the same dose $\left(0.3 \mu \mathrm{C} / \mathrm{cm}^{2}\right)$ to all the grooves to imitate the situation with RIE process and meanwhile varied the groove width as $4 \mu \mathrm{m}, 2 \mu \mathrm{m}, 1 \mu \mathrm{m}$, $0.5 \mu \mathrm{m}$ to investigate the dependence of the resulted groove depth on the groove width. A SEM image of the grating cross section (Figure 5) reveals that the groove depth increases with the groove width, which resembles the lag effect in RIE. This can be attributed to both the cross-linking of the resist and the proximity effect. On the one hand, grooves with small width possess lower resistance to the tension from the neighboring area with high dose, compared with grooves with large width. On the other hand, grooves with small width are more likely to be influenced by the back-scattered electrons from the surrounding areas and subsequently show a high thickness due to over-exposure. Nevertheless, this phenomenon can be avoided by assigning a lower dose to the small width grooves and a higher dose to the large width grooves, thank to the wide dose response range of SU-8. As shown in Figure 6, three $2 \mu \mathrm{m}$-wide grooves ((a1)-(a3)) are assigned with a dose of $0.9 \mu \mathrm{C} / \mathrm{cm}^{2}$, yielding a small groove height that is almost only half compared with that of another group of grooves ((b1)-(b3)) with $1 \mu \mathrm{m}$ width and $0.1 \mu \mathrm{C} / \mathrm{cm}^{2}$ dose. Therefore, the lag effect is totally avoided. Furthermore, one can even obtain grooves with negative depth (i.e. convex ribs) by assigning the groove a dose that is higher than the main structure of the waveguide. Figure 7 shows three $1 \mu \mathrm{m}$-wide ribs $\left(3 \mu \mathrm{C} / \mathrm{cm}^{2}\right)$ together with three $1 \mu \mathrm{m}$ wide grooves $\left(0.1 \mu \mathrm{C} / \mathrm{cm}^{2}\right)$ on the same waveguide $(1.2 \mu \mathrm{C} /$ $\mathrm{cm}^{2}$ ). This can be quite complicated task, if possible at all, in the RIE process. However, it can be realized with high accuracy and fast speed with gray scale EBL.

With the examples above, it is easy to see that groove depths are independent of groove widths in gray scale EBL process, namely the lag effect does not exist anymore. One enjoys both the freedom of depth/width combination of grating trenches and precise control of the dimensions since the dose can be adjusted continuously. To demonstrate the flexibility of the technique in real grating fabrication, we make two set of gratings with variable groove depth and same groove 


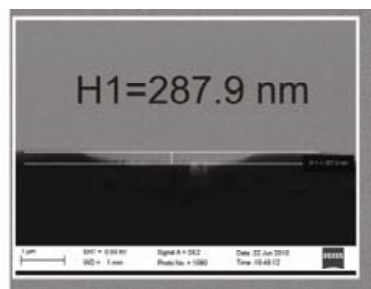

(1)

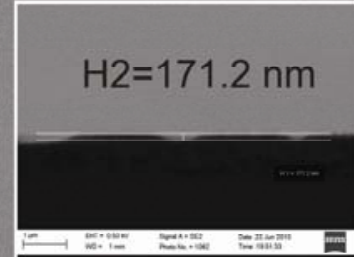

(2)

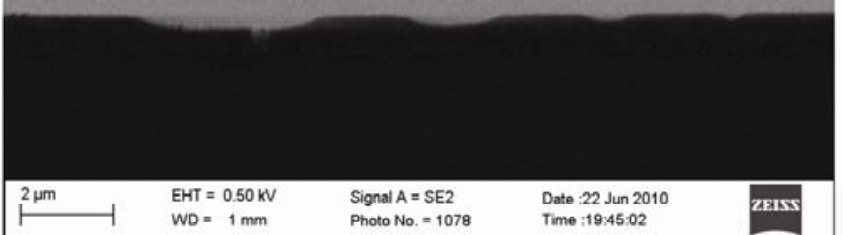

FIG. 5 SEM image of four grooves (cross section view) with the same dose $(0.3 \mu \mathrm{C} /$ $\mathrm{cm}^{2}$ ) but different widths of (1) $4 \mu \mathrm{m}$, (2) $2 \mu \mathrm{m}$, (3) $1 \mu \mathrm{m}$, and (4) $0.5 \mu \mathrm{m}$. The dose for the main structure of the waveguide is $2.4 \mu \mathrm{C} / \mathrm{cm}^{2}$. The PEB condition is $105^{\circ} \mathrm{C}$ for $1 \mathrm{~min}$. It shows that the depth of the groove increases with the width of it (the insets show the depth of groove (1) is $287.9 \mathrm{~nm}$ and the depth of groove (3) $171.2 \mathrm{~nm}$ ), provided the dose is kept constant, which resembles the lag-effect in RIE etching.

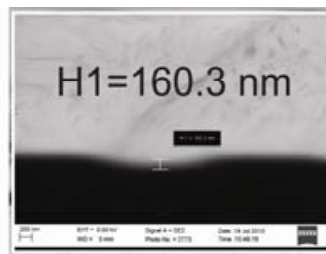

(a1)

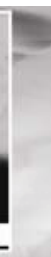

(a2)

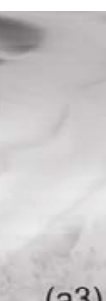

(a3)

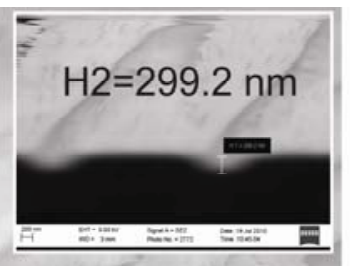

(b1) (b2) (b3)

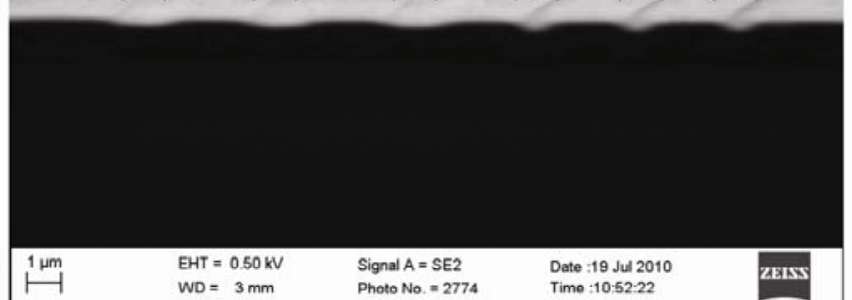

FIC. 6 SEM image of grooves ((a1)-(a3), cross section view) with large width ( $2 \mu \mathrm{m})$ and high dose $\left(0.9 \mu \mathrm{C} / \mathrm{cm}^{2}\right)$, together with grooves ((b1)-(b3) with small width $(1 \mu \mathrm{m})$ and low dose $\left(0.1 \mu \mathrm{C} / \mathrm{cm}^{2}\right)$. The dose for the main structure of the waveguide is $2.4 \mathrm{\mu C} / \mathrm{cm}^{2}$. The post exposure baking is taken under $105^{\circ} \mathrm{C}$ for $1 \mathrm{~min}$. The insets

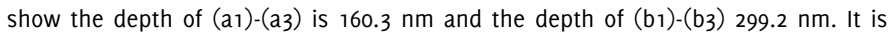
demonstrated that by a carefully designed dose assignment, an anti-lag situation can be achieved (smaller groove width with larger groove depth).

width. The SEM images of the cross section for the two gratings are shown separately in Figures 8a) and 8c). There are ten grooves in Figure 8a) with linearly increased dose from 0.5 to $2.3 \mu \mathrm{C} / \mathrm{cm}^{2}$ and the same width of $650 \mathrm{~nm}$. The dose for the main waveguide is $2.4 \mu \mathrm{C} / \mathrm{cm}^{2}$ (inset of Figure $8 \mathrm{a}$ )). The other set of grating in Figure 8c) has the same dose distribution and width for ten grooves but a smaller dose of $1.2 \mu \mathrm{C} /$ $\mathrm{cm}^{2}$ for the waveguide (inset of Figure $8 \mathrm{c}$ )). To eliminate the

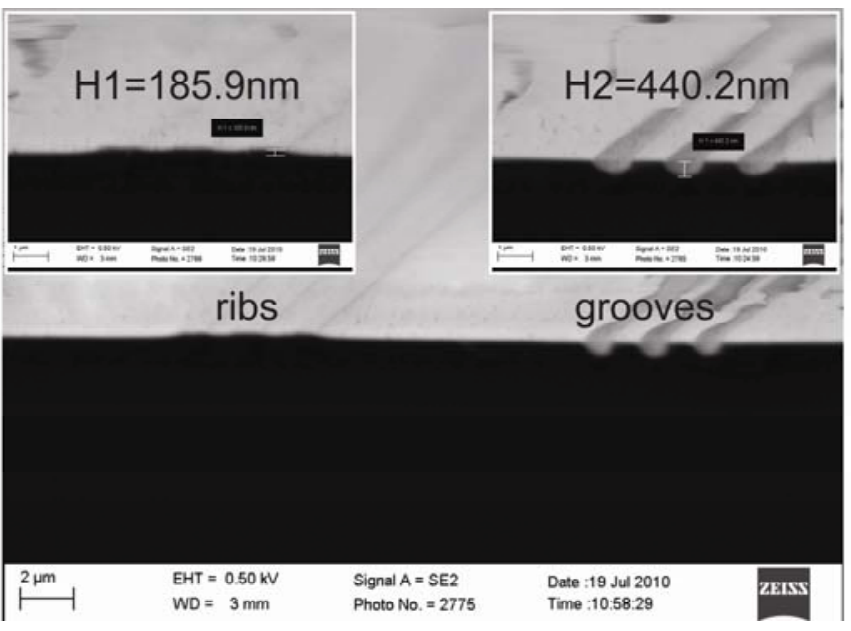

FIG. 7 SEM image of ribs (cross section view) with width of $1 \mu \mathrm{m}$ and dose of $3 \mu \mathrm{C}$ / $\mathrm{cm}^{2}$, together with grooves with width of $1 \mu \mathrm{m}$ and dose of $0.1 \mu \mathrm{C} / \mathrm{cm}^{2}$. The dose for the main structure of the waveguide is $1.2 \mu \mathrm{C} / \mathrm{cm}^{2}$. The PEB condition is $105^{\circ} \mathrm{C}$ for $1 \mathrm{~min}$. The insets show the height the ribs is $185.9 \mathrm{~nm}$ and the depth of the grooves $440.2 \mathrm{~nm}$. It demonstrates the potential to make complex output coupling grating scheme utilizing the gray scale EBL.

vision unclearness of the groove depth induced by the scaling, we also take atomic force microscopy (AFM) images of the two gratings (Figures $8 b$ ) and $8 d$ )) with proper scale adjustment of the two dimensions. Both the SEM and AFM images show that the profiles of the gratings follow well with the applied dose profiles (inset of Figures 8a) and 8c)), while the width of the grooves are kept constant. Specifically, the groove depth of the second set of grating, measured by AFM, versus the exposure dose is plotted in Figure 9. A linear increase of the groove depth with the dose is observed between 1 and $2 \mu \mathrm{C} / \mathrm{cm}^{2}$, verifying the result in Section 3.1. It demonstrates that the linearity of the groove depths within this dose range is valid not only in free-standing structures (Figure 1) but also in real output coupler prototypes with waveguide integrated (Figure 8c)). The influence by the waveguide is negligible. Therefore, this dose range can be utilized to have precise control of the groove profiles.

\section{CONCLUSION}

We investigated the sensitivity and contrast response of SU-8 in the range of low exposure dose for gray scale electron beam lithography to fabricate waveguide and grating output couplers using polymer material. The dose less than $1.6 \mu \mathrm{C} / \mathrm{cm}^{2}$ with $105{ }^{\circ} \mathrm{C}$ post exposure bake is found to be suitable for waveguide writing, while the dose ranges from 1 to $2 \mu \mathrm{C} /$ $\mathrm{cm}^{2}$ for groove depth control. A shrinking effect may affect the accuracy of fabrication for isolated structures with nanoscale features. On the other hand, it can be diminished by a larger total pattern size with same features. As an example, we demonstrated simple and accurate prototyping of polymer based waveguide and grating output coupler with fixed 
a)
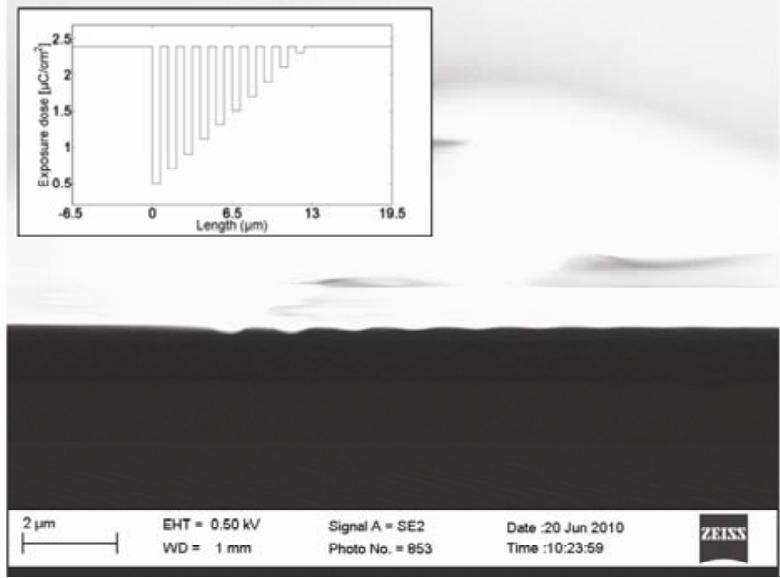

c)

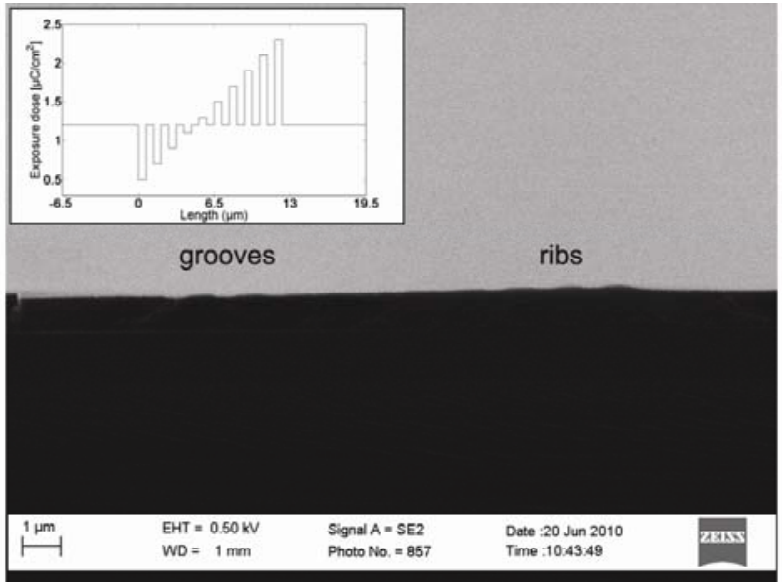

b)

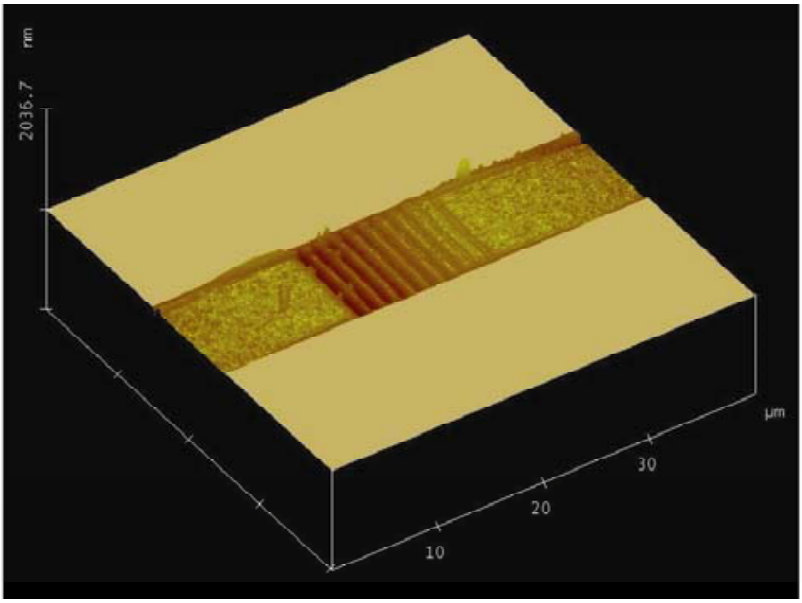

d)

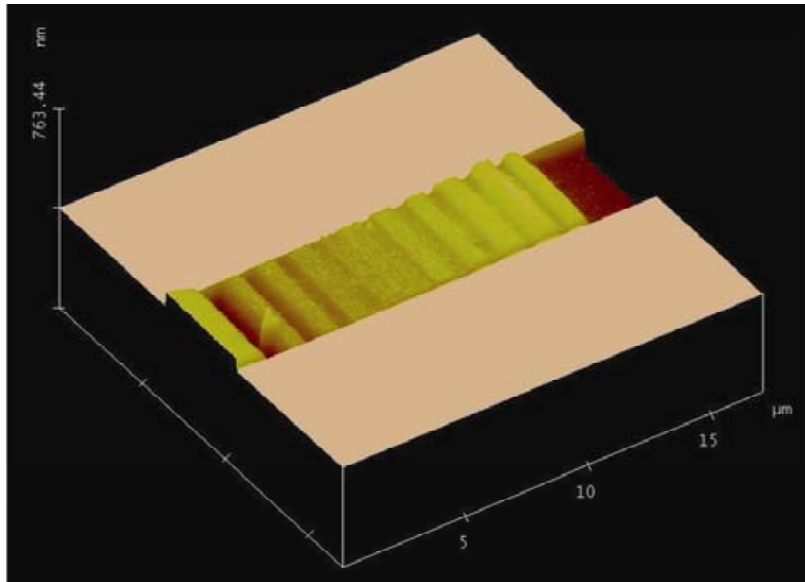

FIG. 8 SEM images of a 10-period SU-8 grating (cross section view) with variable groove depth on a waveguide with a) 2.4 and $\mathrm{c}$ ) $1.2 \mu \mathrm{C} / \mathrm{cm}^{2}$ dose. The groove widths are $650 \mathrm{~nm}$ for both. The PEB condition is $105^{\circ} \mathrm{C}$ for $1 \mathrm{~min}$. The insets show the grooves dose assignment for the two gratings, respectively. AFM images of structures in a) and c) are shown in b) and d), respectively. Because the depth of the grooves is much smaller than the dimensions in the horizontal direction, the scales of the coordinate in the AFM images are readjusted to give a more intuitive view of the groove profile. For the grating shown in c), grooves with larger dose than the main waveguide turn out to be ribs.

or variable groove depth using the gray scale EBL. The lag effect (persistent for reactive ion etching with fabricating of SOI structures) is totally avoided with this technique. It allows free combination of groove width, depth and shape.

The 3D polymer component made with gray scale EBL reveal certain advantages in comparison with SOI structure of similar class: its fabrication is faster, simpler, and cheaper, which is essentially important for research and prototyping purpose, where a lithography mask is typically used only once; this technique provides better accuracy in matching various components with different relief depth since one-stage process does not require additional alignment unavoidable in multi-stage fabrication; finally, fabrication of structures with arbitrary variable depth cannot be realized in one-stage process for SOI components, where reactive ion etching is involved.

Summarizing, this is a promising tool for manufacturing 3D polymer gratings, nanoimprinting stamps and nanophotonics devices.

\section{References}

[1] J. Hiltunen, M. Hiltunen, J. Puustinen, J. Lappalainen, and P. Kari-

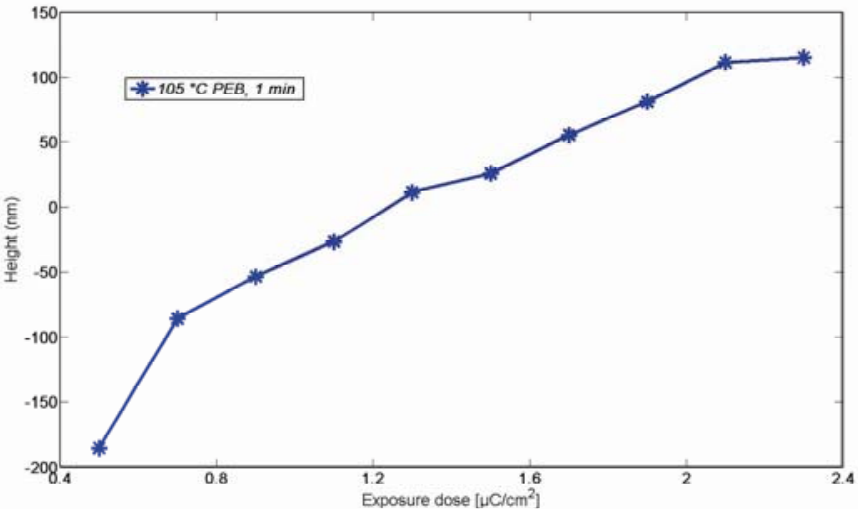

FIG. 9 Groove depths (measured by AFM imaging) for the grating shown in Figures 8 (c) and (d). A linear increase range between 1 and $2 \mu \mathrm{C} / \mathrm{cm}^{2}$ is observed.

oja, "Fabrication of optical waveguides by imprinting: usage of positive tone resist as a mould for UV-curable polymer" Opt. Express 17, 22813-22822 (2009).

[2] S. Ricciardi, S. Popov, A. T. Friberg, and S. Sergeyev, "Tunable microcavity solid-state dye laser for biometrics applications" Proc. SPIE 6729, 67292N (2007). 
[3] D. Nilsson, S. Balslev, M. M. Gregersen, and A. Kristensen, "Micro -fabricated solid state dye lasers based on a photo/definable polymer" Appl. Opt. 44, 4965-4971 (2005).

[4] D. Tailaert, F. Van laere, M. Ayre, W. Bogaerts, D. Van Thourhout, P. Bienstman, and R. Baets, "Grating couplers for coupling between optical fibers and nanophotonic waveguides" Jpn. J. Appl. Phys. 45, 6071-6077 (2006).

[5] S. Scheerlinck, J. Schrauwen, F. Van Laere, D. Van Thourhout, and R. Baets, "Efficient, broadband and compact metal grating couplers for silicon-on-insulator waveguides" Opt. Express 15, 96259630 (2007).

[6] J. S. Yang, C. H. Choi, B. 0, S. G. Lee, and E. Lee, "Design and characterization of an out-of-plane polymer waveguide grating coupler" Proc. SPIE 6476, 647611 (2007).

[7] B. Wang, J. H. Jiang, and G. P. Nordin, "Embedded slanted grating for vertical coupling between fibers and silicon-on-insulator planar waveguides" IEEE Photon. Technol. Lett. 17, 1884-1886 (2005).

[8] V. Kudryashov, X. C. Yuan, W. C. Cheong, and K. Radhakrishnan, "Grey scale structures formation in SU-8 with e-beam and UV" Microelectron. Eng. 67-68, 306-311 (2003).

[9] W. H. Wong, and E. Y. B. Pun, "Exposure characteristics and three -dimensional profiling of SU8C resist using electron beam lithography" J. Vac. Sci. Technol. B 19, 732-735 (2001).
[10] E. A. Shields, F. Williamson, and J. R. Leger, "Electron-beam lithography for thick refractive optical elements in SU-8" J. Vac. Sci. Technol. B 21, 1453-1458 (2003).

[11] K. A. Bates, L. Li, R. L. Roncone, J. Burke, "Gaussian beams from variable groove depth grating couplers in planar waveguides" Appl Opt. 32, 2112-2116 (1993).

[12] H. Jansen, M. D. Boer, R. Wiegerink, N. Tas, E. Smulders, C. Neagu, and M. Elwenspoek, "RIE lag in high aspect ratio trench etching of silicon" Microelectron. Eng. 35, 45-50 (2003).

[13] R. Waldhausl, B. Schnabel, P. Dannberg, E. Kley, A. Brauer, and W. Karthe, "Efficient coupling into polymer waveguides by gratings" Appl. Opt. 36, 9383-9390 (1997).

[14] S. Balslev, T. Rasmussen, P. Shi, and A. Kristensen, "Single mode solid state distributed feedback dye laser fabricated by gray scale electron beam lithography on a dye doped SU-8 resist", J. Micromech. Microeng. 15, 2456-2460 (2005).

[15] P. Rai-choudhury, Handbook of microlithography, micromachining and microfabrication, volume 1: microlithography (SPIE and The Institution of Electrical Engineers, Stevenage, 1997).

[16] A. del Campo, and C. Greiner, "SU-8: a photoresist for highaspect ratio and 3D submicron lithography" J. Micromech. Microeng. 17, R81-R95 (2007). 